\title{
Reactive Systems, Barbed Semantics, and the Mobile Ambients ${ }^{\star}$
}

\author{
Filippo Bonchi ${ }^{1,2}$, Fabio Gadducci ${ }^{1}$, and Giacoma Valentina Monreale ${ }^{1}$ \\ 1 Dipartimento di Informatica, Università di Pisa, Italy \\ 2 Centrum voor Wiskunde en Informatica, Amsterdam, The Netherlands
}

\begin{abstract}
Reactive systems, proposed by Leifer and Milner, represent a meta-framework aimed at deriving behavioral congruences for those specification formalisms whose operational semantics is provided by rewriting rules. Despite its applicability, reactive systems suffered so far from two main drawbacks. First of all, no technique was found for recovering a set of inference rules, e.g. in the so-called SOS style, for describing the distilled observational semantics. Most importantly, the efforts focused on strong bisimilarity, tackling neither weak nor barbed semantics.

Our paper addresses both issues, instantiating them on a calculus whose semantics is still in a flux: Cardelli and Gordon's mobile ambients.

While the solution to the first issue is tailored over our case study, we provide a general framework for recasting (weak) barbed equivalence in the reactive systems formalism. Moreover, we prove that our proposal captures the behavioural semantics for mobile ambients proposed by Rathke and Sobociński and by Merro and Zappa Nardelli.
\end{abstract}

\section{Introduction}

Reactive systems [1] were proposed by Leifer and Milner as an abstract formalism for specifying the dynamics of a computational device. Indeed, the usual specification technique is based on a reduction system: a set, representing the possible states of the device; and a relation among these states, usually inductively defined, representing the possible evolutions of the device. Despite the advantage of conveying the semantics with relatively few compact rewriting rules, freely instantiated and contextualized, the main drawback of reduction-based solutions is poor compositionality, since the dynamic behaviour of arbitrary standalone terms can be interpreted only by inserting them in appropriate contexts, where a reduction may take place. The theoretical appeal of reactive systems is their ability to distill labelled transition systems (LTSs), hence, behavioural equivalences, for devices specified by a reduction system. The idea is simple: whenever a device specified by a term $C[P]$, i.e., by a subterm $P$ inserted into a (unary) context $C[-]$, may evolve to a state $Q$, the associated labelled transition system has a transition $P \stackrel{C[-]}{\longrightarrow} Q$, i.e., the state $P$ evolves into $Q$ with a label $C[-]$.

\footnotetext{
* Partly supported by the EU FP6-IST IP 16004 SEnSOrIA and carried out during
} the first author's tenure of an ERCIM "Alain Bensoussa" Fellowship Programme. 
If all contexts are admitted, the resulting semantics is called saturated semantics, and the resulting strong bisimilarity on the derived LTS is a congruence. Clearly, it becomes unfeasible to check the bisimulation game. Hence, it is necessary to consider a subset of contexts guaranteeing that the distilled behavioural semantics is a congruence. Such a set, the "minimal" contexts allowing a reduction to occur, was identified in [1] by the categorical notion of relative pushout: the resulting strong bisimilarity is a congruence, even if it often does not coincide with the saturated one. Semi-saturated equivalences [23] were introduced for recovering saturated semantics. The bisimulation game becomes asymmetric, and a minimal context may be matched by any context. They capture saturated semantics, yet slashing the number of transitions that need to be checked.

Several attempts were made to encode specification formalisms (Petri nets 4.5, logic programming [2, etc.) as reactive systems, either hoping to recover the standard observational equivalence, whenever such a behavioural semantics exists (CCS [6], pi-calculus [7], etc.), or trying to distill a meaningful semantics. The results are not yet fully satisfactory. On the one-side, bisimilarity via minimal contexts is usually too fine-grained. On the other side, saturated semantics are often too coarse, and for e.g. CCS the standard strong bisimilarity is strictly included in the saturated one. The situation is potentially worse for weak saturated semantics. Intuitively, since in weak semantics the observer can not check for the occurrence of reductions, all systems are observationally equivalent.

Barbs were introduced by Milner and Sangiorgi [8] for addressing this kind of problem. Intuitively, a barb is just a predicate on the states of a system, and barbed equivalences add the check of such predicates in the bisimulation game. The flexibility of the definition allows for recasting a wide variety of observational, bisimulation-based equivalences. Theoretically, the main contribution of our paper is the introduction of suitable notions of barbed and weak barbed saturated semantics for reactive systems, and their characterization via transition systems labelled with minimal contexts, by exploiting the semi-saturated game.

The results above may have potentially far reaching consequences over the usability for the reactive system formalism. However, their adequacy has to be properly established, by checking it against suitable case studies. To this end, we instantiate our proposal over a calculus whose observational semantics is still in a flux, namely, the calculus of mobile ambients, proposed by Cardelli and Gordon [9]. We proceed as follows. First of all, we consider a minimal context semantics for ambients, as distilled in 10 by means of a graphical encoding. Another drawback of reactive systems is that such distilled semantics are usually not in a standard form. So, we propose an alternative, yet equivalent presentation of that LTS, by means of a set of SOS rules. This is a first step toward a possible overcoming of the problem, but here we use such a characterization to establish that the resulting LTS is the same as the one previously proposed by Rathke and Sobociński [11. This is pivotal in proving our main practical result, namely, that barbed and weak barbed semi-saturated semantics for mobile ambients do 
capture the strong and weak barbed congruences for the calculus, as proposed by Rathke and Sobociński [1] and by Merro and Zappa Nardelli [12, respectively.

The paper is organized as follows. Section 2 recalls the basic notions of reactive systems, while Section 3 similarly introduces the main definitions concerning mobile ambients. Section 4 presents the LTS for ambients that we synthesized in [10], introduces a novel characterization of it by means of SOS rules, and finally proves its equivalence with the LTS proposed by Rathke and Sobociński in [11. Section 5 presents the technical core of the paper, the introduction of barbed and weak barbed saturated semantics for reactive systems, and offers a labelled characterization by means of their semi-saturated counterparts. Finally, Section 6 proves that the two barbed semi-saturated bisimilarities we introduced capture the barbed congruences proposed so far for mobile ambients.

\section{Reactive Systems}

This section summarizes the main results concerning (the theory of) reactive systems (RSs) [1]. The formalism aims at deriving labelled transition systems (LTSs) and bisimulation congruences for a system specified by a reduction semantics, and it is centered on the concepts of term, context and reduction rules: contexts are arrows of a category, terms are arrows having as domain 0 , a special object that denotes groundness, and reduction rules are pairs of (ground) terms.

Definition 1 (Reactive System). A reactive system $\mathbb{C}$ consists of

1. a category $\mathbf{C}$;

2. a distinguished object $0 \in|\mathbf{C}|$;

3. a composition-reflecting subcategory $\mathbf{D}$ of reactive contexts;

4. a set of pairs $\mathfrak{R} \subseteq \bigcup_{I \in|\mathbf{C}|} \mathbf{C}(0, I) \times \mathbf{C}(0, I)$ of reduction rules.

Intuitively, reactive contexts are those in which a reduction can occur. By composition-reflecting we mean that $d^{\prime} \circ d \in \mathbf{D}$ implies $d, d^{\prime} \in \mathbf{D}$. Note that the rules have to be ground, i.e., left-hand and right-hand sides have to be terms without holes and, moreover, with the same codomain.

The reduction relation is generated from the reduction rules by closing them under all reactive contexts. Formally, the reduction relation is defined by taking $P \rightsquigarrow Q$ if there is $\langle l, r\rangle \in \mathfrak{R}$ and $d \in \mathbf{D}$ such that $P=d \circ l$ and $Q=d \circ r$.

Thus the behaviour of a $\mathrm{RS}$ is expressed as an unlabelled transition system. In order to obtain a LTS, we can plug a term $P$ into some context $C[-]$ and observe if a reduction occurs. In this case we have that $P \stackrel{C[-]}{\longrightarrow}$. Categorically speaking, this means that $C[-] \circ P$ matches $d \circ l$ for some rule $\langle l, r\rangle \in \mathfrak{R}$ and some reactive context $d$. This situation is formally depicted by diagram (i) in Fig. 1] a commuting diagram like this is called a redex square.

Definition 2 (Saturated Transition System). The saturated transition system (STS) is defined as follows

- states: arrows $P: 0 \rightarrow I$ in $\mathbf{C}$, for arbitrary $I$;

- transitions: $P \stackrel{C[-]}{\rightarrow}{ }_{S A T} Q$ if $C[P] \rightsquigarrow Q$. 


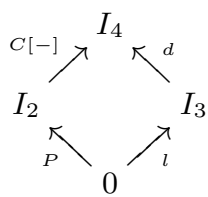

(i)

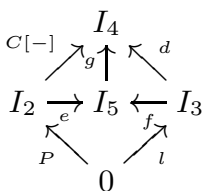

(ii)

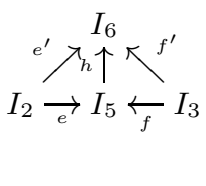

(iii)

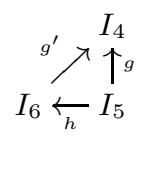

(iv)

Fig. 1. Redex Square and RPO

Note that $C[P]$ is a stand-in for $C[-] \circ P$ : the same notation is chosen in Definitions 3 and 6 below, in order to allows for an easier comparison with the process calculi notation, to be adopted in the following sections.

Definition 3 (Saturated Bisimulation). Saturated bisimilarity $\sim^{S}$ is the largest symmetric relation $\mathcal{R}$ such that whenever $P \mathcal{R} Q$ then $\forall C[-]$

- if $C[P] \rightsquigarrow P^{\prime}$ then $C[Q] \rightsquigarrow Q^{\prime}$ and $P^{\prime} \mathcal{R} Q^{\prime}$.

It is obvious that $\sim^{S}$ is a congruence. Indeed, it is the coarsest symmetric relation satisfying the bisimulation game on $\rightsquigarrow$ that is also a congruence.

Note that STS is often infinite-branching since all contexts allowing reductions may occur as labels. Moreover, it has redundant transitions. For example, consider the term $a .0$ of CCS. We have both the transitions $a .0 \stackrel{\bar{a} .0 \mid-}{\rightarrow} S A T 0 \mid 0$ and

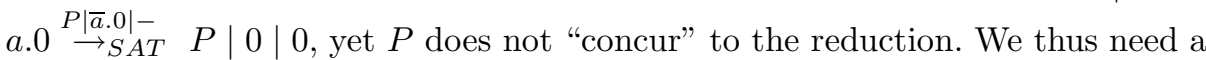
notion of "minimal context allowing a reduction", captured by idem pushouts.

Definition 4 (RPO, IPO). Let the diagrams in Fig. 1] be in a category $\mathbf{C}$, and let $(i)$ be a commuting diagram. A candidate for $(i)$ is any tuple $\left\langle I_{5}, e, f, g\right\rangle$ making (ii) commute. A relative pushout (RPO) is the smallest such candidate, i.e., such that for any other candidate $\left\langle I_{6}, e^{\prime}, f^{\prime}, g^{\prime}\right\rangle$ there exists a unique morphism $h: I_{5} \rightarrow I_{6}$ making (iii) and (iv) commute. A commuting square such as diagram (i) of Fig. 1 is called idem pushout (IPO) if $\left\langle I_{4}, c, d, i d_{I_{4}}\right\rangle$ is its RPO.

Hereafter, we say that a $\mathrm{RS}$ has redex RPOs (IPOs) if every redex square has an RPO (IPO) as candidate. For a better understanding of these two notions, we refer the reader to [2]. For the aim of this paper it is enough to know that the former notion is more restrictive than the latter.

Definition 5 (IPO-Labelled Transition System). The IPO-labelled transition system (ITS) is defined as follows

- states: $P: 0 \rightarrow I$ in $\mathbf{C}$, for arbitrary $I$;

- transitions: $P \stackrel{C}{\rightarrow}{ }_{I P O}^{[-]} d \circ r$ if $d \in \mathbf{D},\langle l, r\rangle \in \mathfrak{R}$, and (i) in Fig. 1 is an IPO.

In other words, if inserting $P$ into the context $C[-]$ matches $d \circ l$, and $C[-]$ is the "smallest" such context, then $P$ transforms to $d \circ r$ with label $C[-]$.

Bisimilarity on ITS is referred to as IPO-bisimilarity $\left(\sim^{I}\right)$. Leifer and Milner have shown that if the RS has redex RPOs, then it is a congruence. 
Proposition 1. In a reactive system having redex-RPOs, $\sim^{I}$ is a congruence.

Clearly, $\sim^{I} \subseteq \sim^{S}$. In 2]3] the first author, with König and Montanari, shows that this inclusion is strict for many formalisms and introduces semi-saturation.

Definition 6 (Semi-Saturated Bisimulation). Semi-saturated bisimilarity $\sim^{S S}$ is the largest symmetric relation $\mathcal{R}$ such that whenever $P \mathcal{R} Q$ then

$$
\text { - if } P \stackrel{C[-]}{\rightarrow}{ }_{I P O}^{\prime} P^{\prime} \text { then } C[Q] \rightsquigarrow Q^{\prime} \text { and } P^{\prime} \mathcal{R} Q^{\prime} \text {. }
$$

Proposition 2. In a reactive system having redex-IPOs, $\sim S S=\sim^{S}$.

\section{Mobile Ambients}

This section shortly recalls the finite, communication-free fragment of mobile ambients (MAs) 9], its reduction semantics and behavioural equivalences.

Fig. 2 shows the syntax of the calculus. We assume a set $\mathcal{N}$ of names ranged over by $m, n, u, \ldots$ Besides the standard constructors, we included a set of process variables $\mathcal{X}=\{X, Y, \ldots\}$, and a set of name variables $\mathcal{V}=\{x, y, \ldots\}$. Intuitively, an extended process $x[P] \mid X$ represents an underspecified process, where either the process $X$ or the name of the ambient $x[-]$ can be further instantiated. These are needed for the presentation of the LTSs in Section 4 .

We let $P, Q, R, \ldots$ range over the set $\mathcal{P}$ of pure processes, containing neither process nor name variables; while $P_{\epsilon}, Q_{\epsilon}, R_{\epsilon}, \ldots$ range over the set $\mathcal{P}_{\epsilon}$ of wellformed processes, i.e., such that no process or ambient variable occurs twice.

We use the standard definitions for the set of free names of a pure process $P$, denoted by $f n(P)$, and for $\alpha$-convertibility, with respect to the restriction operators $(\nu n)$. We moreover assume that $f n(X)=f n(x[\mathbf{0}])=\emptyset$. We also consider a family of substitutions, which may replace a process/name variable with a pure process/name, respectively. Substitutions avoid name capture: for a pure process $P$, the expression $(\nu n)(\nu m)(X \mid x[\mathbf{0}])\left\{m / x,{ }^{n[P]} / X\right\}$ corresponds to the pure process $(\nu p)(\nu q)(n[P] \mid m[\mathbf{0}])$, for names $p, q \notin\{m\} \cup f n(n[P])$.

The semantics of the calculus exploits a structural congruence, denoted by $\equiv$, which is the least equivalence on pure processes that satisfies the axioms shown in Fig. 3. We assume that the structural congruence defined on processes over the extended syntax is induced by the same set of rules shown in Fig. 3.

The reduction relation, denoted by $\rightsquigarrow$, describes the evolution of pure processes over time. It is the smallest relation closed under the congruence $\equiv$ and inductively generated by the set of axioms and inference rules shown in Fig. 4 ,

A strong barb $o$ is a predicate over the states of a system, with $P \downarrow_{o}$ denoting that $P$ satisfies $o$. In MAs, $P \downarrow_{n}$ denotes the presence at top-level of a unrestricted ambient $n$. Formally, for a pure process $P, P \downarrow_{n}$ if $P \equiv(\nu A)(n[Q] \mid R)$ and $n \notin A$, for some processes $Q$ and $R$ and a set of restricted names $A$. A pure process $P$ satisfies the weak barb $n$ (denoted as $P \Downarrow_{n}$ ) if there exists a process $P^{\prime}$ such that $P \rightsquigarrow^{*} P^{\prime}$ and $P^{\prime} \downarrow_{n}$, where $\rightsquigarrow^{*}$ is the transitive and reflexive closure of $\rightsquigarrow$. 
Fig. 2. (Extended) Syntax of mobile ambients

\begin{tabular}{ll}
\hline if $P \equiv Q$ then $P|R \equiv Q| R$ & $P \mid \mathbf{0} \equiv P$ \\
if $P \equiv Q$ then $(\nu n) P \equiv(\nu n) Q$ & $(\nu n)(\nu m) P \equiv(\nu m)(\nu n) P$ \\
if $P \equiv Q$ then $n[P] \equiv n[Q]$ & $(\nu n)(P \mid Q) \equiv P \mid(\nu n) Q \quad$ if $n \notin f n(P)$ \\
if $P \equiv Q$ then $M . P \equiv M . Q$ & $(\nu n) m[P] \equiv m[(\nu n) P] \quad$ if $n \neq m$ \\
$P|Q \equiv Q| P$ & $(\nu n) M . P \equiv M .(\nu n) P \quad$ if $n \notin f n(M)$ \\
$(P \mid Q)|R \equiv P|(Q \mid R)$ & $(\nu n) P \equiv(\nu m)(P\{m / n\})$ if $m \notin f n(P)$ \\
\hline
\end{tabular}

Fig. 3. Structural congruence

\begin{tabular}{ll}
\hline$n[$ in $m . P \mid Q] \mid m[R] \rightsquigarrow m[n[P \mid Q] \mid R]$ & if $P \rightsquigarrow Q$ then $(\nu n) P \rightsquigarrow(\nu n) Q$ \\
$m[n[$ out $m . P \mid Q] \mid R] \rightsquigarrow n[P \mid Q] \mid m[R]$ & if $P \rightsquigarrow Q$ then $n[P] \rightsquigarrow n[Q]$ \\
open $n . P|n[Q] \rightsquigarrow P| Q$ & if $P \rightsquigarrow Q$ then $P|R \rightsquigarrow Q| R$ \\
\hline
\end{tabular}

Fig. 4. Reduction relation on pure processes

Definition 7 (Reduction Barbed Congruences [11 12]). Strong reduction barbed congruence $\cong$ is the largest symmetric relation $\mathcal{R}$ such that whenever $P \mathcal{R} Q$ then

- if $P \downarrow_{n}$ then $Q \downarrow_{n}$;

- if $P \rightsquigarrow P^{\prime}$ then $Q \rightsquigarrow Q^{\prime}$ and $P^{\prime} \mathcal{R} Q^{\prime}$;

- $\forall C[-], C[P] \mathcal{R} C[Q]$.

Weak reduction barbed congruence $\cong W$ is the largest symmetric relation $\mathcal{R}$ such that whenever $P \mathcal{R} Q$ then

- if $P \downarrow_{n}$ then $Q \Downarrow_{n}$;

- if $P \rightsquigarrow P^{\prime}$ then $Q \rightsquigarrow{ }^{*} Q^{\prime}$ and $P^{\prime} \mathcal{R} Q^{\prime}$;

- $\forall C[-], C[P] \mathcal{R} C[Q]$.

Labelled characterization of reduction barbed congruences over MAs processes are presented by Rathke and Sobociński for the strong case [11, and by Merro and Zappa Nardelli for the weak one [12].

The main result of this paper is the proposal of a novel notion of barbed saturated bisimilarity over reactive systems, both for the strong and weak case, that is able to capture the two behavioural semantics for MAs defined above.

\section{Labelled Transition Systems for Mobile Ambients}

In this section we first recall the IPO-transition system for MAs, previously proposed in [10], and then we introduce a SOS presentation for it. Finally, we 
(TAU) $\frac{P \rightsquigarrow Q}{P \rightrightarrows Q}$

(IN) $\frac{P \equiv(\nu A)\left(\text { in } m . P_{1} \mid P_{2}\right) \quad m \notin A}{P \stackrel{x-\left[-\mid X_{1}\right] \mid m\left[X_{2}\right]}{\longrightarrow}(\nu A) m\left[x\left[P_{1}\left|P_{2}\right| X_{1}\right] \mid X_{2}\right]}$

(INAmB) $\frac{P \equiv(\nu A)\left(n\left[\text { in } m . P_{1} \mid P_{2}\right] \mid P_{3}\right) \quad m \notin A}{P-\stackrel{\mid m\left[X_{1}\right]}{\longrightarrow}(\nu A)\left(m\left[n\left[P_{1} \mid P_{2}\right] \mid X_{1}\right] \mid P_{3}\right)}$

$(\mathrm{CoIN}) \frac{P \equiv(\nu A)\left(m\left[P_{1}\right] \mid P_{2}\right) \quad m \notin A}{P \stackrel{-\mid x\left[i n m \cdot X_{1} \mid X_{2}\right]}{\longrightarrow}(\nu A)\left(m\left[x\left[X_{1} \mid X_{2}\right] \mid P_{1}\right] \mid P_{2}\right)}$
(OUT) $\frac{P \equiv(\nu A)\left(\text { out } m . P_{1} \mid P_{2}\right) \quad m \notin A}{P \stackrel{m\left[x\left[-\mid X_{1}\right] \mid X_{2}\right]}{\longrightarrow}(\nu A)\left(m\left[X_{2}\right] \mid x\left[P_{1}\left|P_{2}\right| X_{1}\right]\right)}$

(OutAmB) $\frac{P \equiv(\nu A)\left(n\left[\text { out } m . P_{1} \mid P_{2}\right] \mid P_{3}\right) \quad m \notin A}{P \stackrel{m\left[-\mid X_{1}\right]}{\longrightarrow}(\nu A)\left(m\left[P_{3} \mid X_{1}\right] \mid n\left[P_{1} \mid P_{2}\right]\right)}$

(OPEN) $\frac{P \equiv(\nu A)\left(\text { open } n . P_{1} \mid P_{2}\right) \quad n \notin A}{P \stackrel{-\mid n\left[X_{1}\right]}{\longrightarrow}(\nu A)\left(P_{1}\left|P_{2}\right| X_{1}\right)}$

$($ CoOpen $) \frac{P \equiv(\nu A)\left(n\left[P_{1}\right] \mid P_{2}\right) \quad n \notin A}{P=\underline{-\operatorname{lopen} n \cdot X_{1}}(\nu A)\left(P_{1}\left|X_{1}\right| P_{2}\right)}$

Fig. 5. The LTS $\mathcal{D}$

discuss the relationship between our SOS LTS and the LTS for MAs proposed by Rathke and Sobociński in [11. Note that we implicitly assume that all the LTSs that we define are closed with respect to structural congruence.

\subsection{An IPO-LTS for Mobile Ambients}

This section presents the ITS $\mathcal{D}$ for MAs proposed in [10. The inference rules describing this LTS are obtained from an analysis of a LTS over (processes as) graphs, derived by the borrowed context mechanism [13, which is an instance of the theory of RSs [14]. The labels of the transitions are unary contexts, i.e., terms of the extended syntax with a hole - . Note that they are minimal contexts, that is, they represent the exact amount of context needed by a system to react. We denote them by $C_{\epsilon}[-]$. The formal definition of the LTS is presented in Fig. 5 ,

The rule TAU represents the $\tau$-actions modeling internal computations. Notice that the labels of the transitions are identity contexts composed of just a hole -, while the resulting states are processes over MAs standard syntax.

The other rules in Fig. 5 model the interactions of a process with its environment. Note that both labels and resulting states contain process and name variables. We define the $\operatorname{LTS} \mathcal{D}_{\mathcal{I}}$ for processes over the standard syntax of MAs by instantiating all the variables of the labels and of the resulting states.

Definition 8. Let $P, Q$ be pure processes and let $C[-]$ be a pure context. Then, we have that $P \stackrel{C[-]}{\longrightarrow} \mathcal{D}_{\mathcal{I}} Q$ if there exists a transition $P \stackrel{C_{\epsilon}[-]}{\longrightarrow} \mathcal{D} Q_{\epsilon}$ and a substitution $\sigma$ such that $Q_{\epsilon} \sigma=Q$ and $C_{\epsilon}[-] \sigma=C[-]$.

In the above definition we implicitly consider only ground substitutions. Moreover, we recall that the substitutions do not capture bound names.

The rule OpEn models the opening of an ambient provided by the environment. In particular, it enables a process $P$ with a capability open $n$ at top level, for $n \in f n(P)$, to interact with a context providing an ambient $n$ containing some process $X_{1}$. Note that the label $-\mid n\left[X_{1}\right]$ of the rule represents the minimal context needed by the process $P$ for reacting. The resulting state is the process over the extended syntax $(\nu A)\left(P_{1}\left|X_{1}\right| P_{2}\right)$, where $X_{1}$ represents a process provided by the environment. Note that the instantiation of the process variable $X_{1}$ 
with a process containing a free name that belongs to the bound names in $A$ is possible only $\alpha$-converting the resulting process $(\nu A)\left(P_{1}\left|X_{1}\right| P_{2}\right)$ into a process that does not contain that name among its bound names at top level.

The rule CoOpen instead models an environment that opens an ambient of the process. The rule INAMB enables an ambient of the process to migrate into a sibling ambient provided by the environment, while in the rule IN both the ambients are provided by the environment. In the rule CoIN an ambient provided by the environment enters an ambient of the process. The rule OutAmB models an ambient of the process exiting from an ambient provided by the environment, while in the rule OuT both ambients are provided by the environment.

The LTS $\mathcal{D}$ does not conform to the so-called SOS style [15: indeed, the premises of the inference rules are just constraints over the structure of the process, as typical of the LTSs distilled by IPOs. In the next section we propose an alternative, yet equivalent set of rules in SOS style, which allows an easier comparison between our proposal and the one by Rathke and Sobociński.

\subsection{A SOS Presentation for the IPO-LTS}

This section proposes a SOS presentation for the ITS $\mathcal{D}$ shown in the previous section. The SOS LTS $\mathcal{S}$ is directly obtained from the LTS $\mathcal{D}$ and it coincides with this last one. The rules of the LTS $\mathcal{S}$ are shown in Fig. 6 ,

The rules in the first two rows of Fig. 6 model internal computations. They are indeed obtained from the first rule in Fig. 5. In particular, since this last rule exactly derives the same transition relation of the reduction relation over MAs, we replace it with the reduction rules labelled with the identity context - . So, we obtain the axioms modelling the execution of the capabilities of the calculus, and a structural rule for each ambient, parallel and restriction operators.

Also the remaining rules in Fig. 6, modelling the interactions of a process with its environment, are obtained from the other rules in Fig. 5 . In particular, for each of these rules we derive three rules. First, we determine the axiom by considering the minimal process needed by the reduction to occur. For e.g. the rule IN of the LTS $\mathcal{D}$, the minimal process allowing the reduction is in m. $P_{1}$. Therefore, we determine the axiom in $m . P_{1} \stackrel{x\left[-\mid X_{1}\right] \mid m\left[X_{2}\right]}{\longrightarrow} m\left[x\left[P_{1} \mid X_{1}\right] \mid X_{2}\right]$. The next step consists in determining the relative structural rules in SOS style. So, as far as the rule IN of the LTS $\mathcal{D}$ is concerned, we have that if $P \stackrel{x\left[-\mid X_{1}\right] \mid m\left[X_{2}\right]}{\longrightarrow} P \epsilon$, then for the process $P \mid Q$ there is a transition labelled $x\left[-\mid X_{1}\right] \mid m\left[X_{2}\right]$ leading to the process $P_{\epsilon}$ with the process $Q$ inside the ambient $x$, that is, $P \mid Q \stackrel{x\left[-\mid X_{1}\right] \mid m\left[X_{2}\right]}{\longrightarrow} P_{\epsilon}\left\{Q \mid X_{1} / X_{1}\right\}$. Instead, if $P \stackrel{x\left[-\mid X_{1}\right] \mid m\left[X_{2}\right]}{\longrightarrow} P \epsilon$ and $m \neq a$, then $(\nu a) P \stackrel{x\left[-\mid X_{1}\right] \mid m\left[X_{2}\right]}{\longrightarrow}(\nu a) P_{\epsilon}$.

Theorem 1. Let $P$ be a pure process and let $C_{\epsilon}[-]$ be a context. Then, $P \stackrel{C_{\epsilon}[-]}{\longrightarrow} \mathcal{D}$ $Q_{\epsilon}$ if and only if $P \stackrel{C_{\epsilon}[-]}{\longrightarrow} \mathcal{S} Q_{\epsilon}$.

As for ITS $\mathcal{D}$, also for LTS $\mathcal{S}$ we define the LTS $\mathcal{S}_{\mathcal{I}}$ for pure processes by instantiating all the variables of the labels and of the resulting states. 


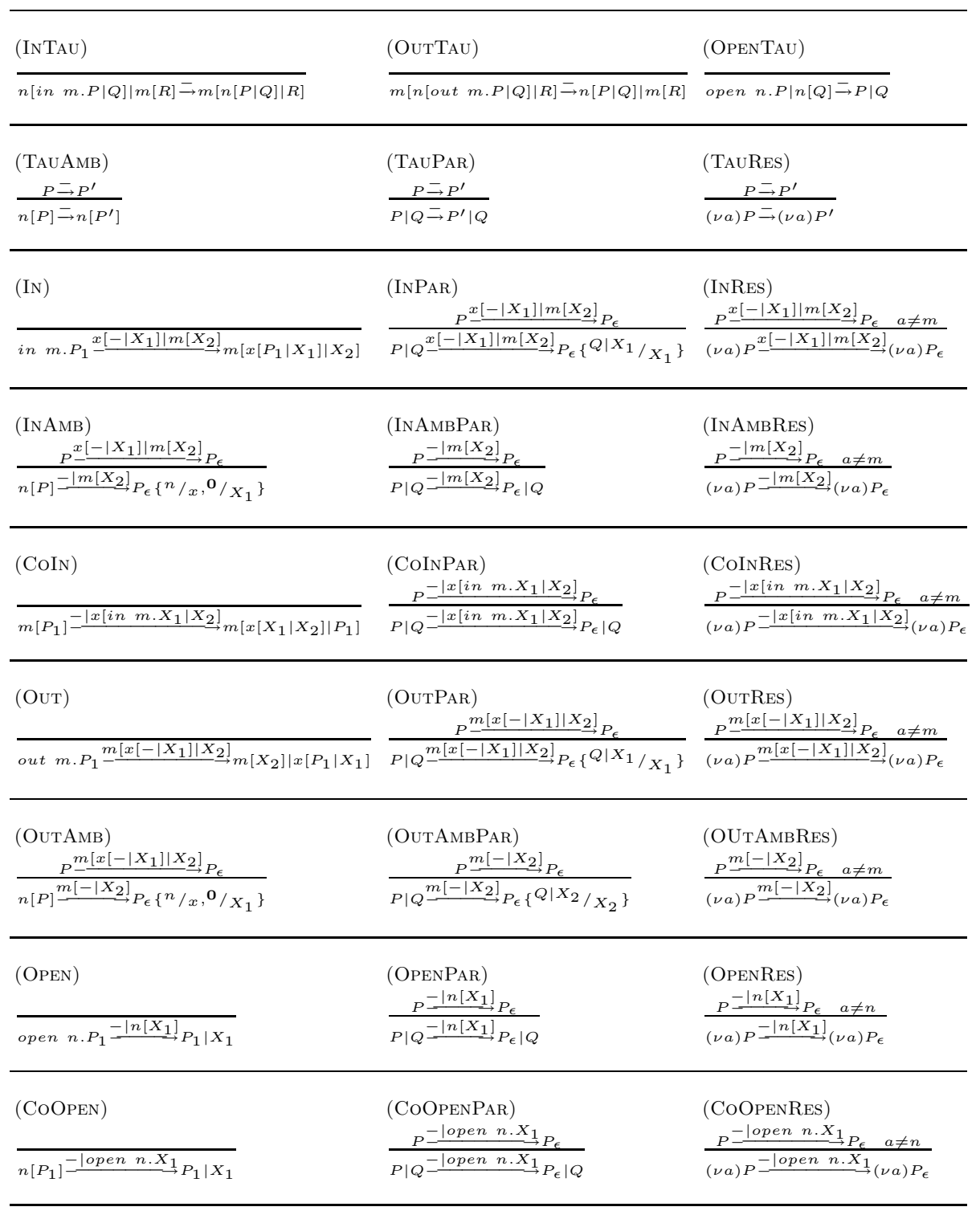

Fig. 6. The LTS $\mathcal{S}$

\subsection{Equivalence between LTSs}

We now show that LTS $\mathcal{S}_{\mathcal{I}}$ defined on processes over the standard syntax of MAs coincides with the LTS for MAs proposed by Rathke and Sobociński in [1]. 


\begin{tabular}{|c|c|c|c|}
\hline$c$ & $C_{\epsilon}^{\alpha}[-]$ & $\alpha$ & $C_{\epsilon}^{\alpha}[-]$ \\
\hline in $m$ & $x\left[-\mid X_{1}\right] \mid m\left[X_{2}\right]$ & {$[$ out $m]$} & $m\left[-\mid X_{2}\right]$ \\
\hline$[$ in $m]$ & $-m\left[X_{2}\right]$ & open $n$ & $-n\left[X_{1}\right]$ \\
\hline$[\overline{i n m}]$ & $-\mid x\left[\right.$ in $\left.m . X_{1} \mid X_{2}\right]$ & $\overline{\text { open } n}$ & - open n. $X_{1}$ \\
\hline out $m$ & $m\left[x\left[-\mid X_{1}\right] \mid X_{2}\right]$ & $\tau$ & - \\
\hline
\end{tabular}

Fig. 7. Correspondence between the labels of the LTS $\mathcal{C}$ and the ones of the LTS $\mathcal{S}$

Their LTS is organized into three components: the process-view LTS $\mathcal{C}$, the context-view LTS $\mathcal{A}$, and the combined LTS $\mathcal{C} \mathcal{A}$. The labels of the LTS $\mathcal{C} \mathcal{A}$ have the shape $\alpha \downarrow \vec{M}$, where $\alpha$ is derived by LTS $\mathcal{C}$, and $\vec{M}$ by LTS $\mathcal{A}$. In a transition $P \stackrel{\alpha \downarrow \vec{M}}{\longrightarrow} \mathcal{C A} Q$, the label $\alpha$ identifies the minimal context needed by the pure process $P$ to react, while $\vec{M}$ is a list of pure processes and ambient names, representing an instantiation of the context components. Therefore, since the labels of our LTS $\mathcal{S}$ are precisely the minimal contexts needed by a system to react, we establish a one-to-one correspondence between the labels $\alpha$ of LTS $\mathcal{C}$ and the labels $C_{\epsilon}[-]$ of our LTS $\mathcal{S}$. Fig. 7 shows this correspondence: $C_{\epsilon}^{\alpha}[-]$ denotes the label of our LTS $\mathcal{S}$ corresponding to the label $\alpha$ of their LTS $\mathcal{C}$.

In the label $\alpha \downarrow \vec{M}$ the list $\vec{M}$ represents an instantiation for the components of the context identified by $\alpha$. Therefore, since the contexts identified by the $\alpha$ 's correspond to the contexts representing our labels, the list $\vec{M}$ of $\alpha \downarrow \vec{M}$ contains as many elements as the variables of the label $C^{\alpha}[-]$. In particular, $\vec{M}$ contains $k$ processes if and only if $k$ process variables occur in $C^{\alpha}[-]$, and analogously, $\vec{M}$ contains $h$ ambient names if and only if $h$ name variables occur in $C^{\alpha}[-]$.

To better understand their relationship, we informally discuss an example showing how a transition in LTS $\mathcal{S}_{\mathcal{I}}$ corresponds to a transition in LTS $\mathcal{C} \mathcal{A}$.

Example 1. Consider the pure process $P=i n m . P_{1}$, for some process $P_{1}$. According to IN rule in Fig. 6, $P \stackrel{x\left[-\mid X_{1}\right] \mid m\left[X_{2}\right]}{\longrightarrow} \mathcal{S} m\left[x\left[P_{1} \mid X_{1}\right] \mid X_{2}\right]$. If we consider a substitution $\sigma=\left\{{ }^{P_{2}} / X_{1},{ }^{n} / x,{ }^{P_{3}} / X_{2}\right\}$, where $P_{2}, P_{3}$ are processes and $n$ is an ambient name, we have $P \stackrel{n\left[-\mid P_{2}\right] \mid m\left[P_{3}\right]}{\longrightarrow} \mathcal{S}_{\mathcal{I}} m\left[n\left[P_{1} \mid P_{2}\right] \mid P_{3}\right]$.

Consider now $\mathcal{C} \mathcal{A}$. According to IN rule of [11], $P \stackrel{\text { in m }}{\longrightarrow} \mathcal{C} \lambda X x Y . m\left[x\left[P_{1} \mid X\right] \mid Y\right]$, where $X, Y$ and $x$ are variables representing the components provided by the context. In particular, $X$ and $Y$ are process variables and $x$ is a name variable. Consider the instantiation for context components induced by substitution $\sigma$, namely, $\vec{M}: P_{2}, n, P_{3}$. According to rule INST of [1], we obtain $\lambda X x Y . m\left[x\left[P_{1} \mid X\right] \mid Y\right] \stackrel{\vec{M} \downarrow}{\longrightarrow} \mathcal{A} m\left[n\left[P_{1} \mid P_{2}\right] \mid P_{3}\right]$ and, by applying $\mathrm{C} \lambda$ rule of [1], $P \stackrel{\text { in } m \downarrow \vec{M}}{\longrightarrow} \mathcal{C A} m\left[n\left[P_{1} \mid P_{2}\right] \mid P_{3}\right]$. Therefore, both LTSs have a transition leading the process $P$ to $m\left[n\left[P_{1} \mid P_{2}\right] \mid P_{3}\right]$.

Theorem 2. Let $P$ be a pure process. If $P \stackrel{\alpha \downarrow \vec{M}}{\longrightarrow} \mathcal{C A} Q$, then there is a unique (up-to $\equiv$ ) substitution $\sigma$ s.t. $P \stackrel{C_{\epsilon}^{\alpha}[-] \sigma}{\longrightarrow} \mathcal{S}_{\mathcal{I}} Q$. Vice versa, if $P \stackrel{C[-]}{\longrightarrow} \mathcal{S}_{\mathcal{I}} Q$, then there are $\alpha$ and a unique (up-to $\equiv$ ) tuple $\vec{M}$ s.t. $C[-]=C^{\alpha}[-]$ and $P \stackrel{\alpha \downarrow \vec{M}}{\longrightarrow} \mathcal{C A} Q$. 


\section{Barbed Semantics for Reactive Systems}

Several attempts were made to encode specification formalisms (Petri nets 415, logic programming [2], CCS [16 17], $\lambda$-calculus [1819], asynchronous $\pi$-calculus 20, fusion calculus [21, etc.) as RSs, either hoping to recover the standard observational equivalences, whenever such a behavioural semantics exists (CCS [6], $\pi$-calculus [7], etc.), or trying to distill a meaningful semantics. Unfortunately, IPO semantics is usually too fine-grained, and MAs are no exception.

On the other hand, saturated semantics are often too coarse. For example, the CCS processes $\omega=\tau . \Omega$ and $\Theta=\tau . \Omega+a . \Omega$ are saturated bisimilar [8, yet not strong bisimilar. This problem becomes potentially serious when considering weak semantics. Intuitively, two systems are saturated bisimilar if they cannot be distinguished by an external observer that, in any moment of their execution, can insert them into some context and observe a reduction. However, since in weak semantics reductions cannot be observed, all systems are equivalent.

Barbs were introduced for overcoming these problems 8 . This section proposes a notion of barbed saturated bisimilarity for RSs, showing that it is efficiently characterized through the IPO-transition systems by exploiting the semi-saturated game: Section 5.1 studies the strong case; Section 5.2, the weak one.

\subsection{Barbed Saturated Bisimilarity}

Barbed bisimilarity was introduced for CCS [8], using barbs $\downarrow_{a}$ and $\downarrow_{\bar{a}}$ that represented the ability of a process to perform an input, respectively an output, on channel $a$. As for MAs, $\downarrow_{n}$ means that an ambient $n$ occurs at top level.

More generally, barbs are predicates over the states of a system. We then fix in the following a family $O$ of barbs, and we write $P \downarrow_{o}$ if $P$ satisfies $o \in O$.

Definition 9 (Barbed Bisimilarity, Barbed Congruence). A symmetric relation $\mathcal{R}$ is a barbed bisimulation if whenever $P \mathcal{R} Q$ then

- if $P \downarrow_{o}$ then $Q \downarrow_{o}$;

- if $P \rightsquigarrow P^{\prime}$ then $Q \rightsquigarrow Q^{\prime}$ and $P^{\prime} \mathcal{R} Q^{\prime}$.

Barbed bisimilarity $\sim^{B}$ is the largest barbed bisimulation; barbed congruence $\simeq^{B}$ is the largest congruence contained in $\sim^{B}$.

Barbed congruence is clearly a congruence, but there is no guarantee that it is also a bisimulation. In this paper, we consider a different notion of behavioural equivalence that is both a bisimulation and a congruence.

Definition 10 (Barbed Saturated Bisimulation). A symmetric relation $\mathcal{R}$ is a barbed saturated bisimulation if whenever $P \mathcal{R} Q$ then $\forall C[-]$

- if $C[P] \downarrow_{o}$ then $C[Q] \downarrow_{o}$;

- if $C[P] \rightsquigarrow P^{\prime}$ then $C[Q] \rightsquigarrow Q^{\prime}$ and $P^{\prime} \mathcal{R} Q^{\prime}$.

Barbed saturated bisimilarity $\sim^{B S}$ is the largest barbed saturated bisimulation. 
It is easy to see that $\sim^{B S}$ is the largest barbed bisimulation that is also a congruence, and that it is finer than $\simeq^{B}$ (the largest congruence contained into barbed bisimilarity). Intuitively, in the former case the external observer can plug systems into contexts at any step of their execution, while in the latter the observer can contextualize systems only at the beginning. The former observer is more powerful than the latter, thus proving that $\sim^{B S}$ is indeed finer than $\simeq^{B}$.

It is our opinion that $\sim^{B S}$ is more appropriate, in order to model concurrent interactive systems embedded in an environment that continuously changes. And while in several formalisms the two notions coincide [22, for MAs the standard behavioural equivalence $\cong$ (Definition 7) is clearly an instance of $\sim^{B S}$.

Most importantly, though, barbed saturated bisimilarity can be efficiently characterized through the IPO-transition system via the semi-saturated game.

Definition 11 (Barbed Semi-Saturated Bisimulation). A symmetric relation $\mathcal{R}$ is a barbed semi-saturated bisimulation if whenever $P \mathcal{R} Q$ then

$-\forall C[-]$, if $C[P] \downarrow_{o}$ then $C[Q] \downarrow_{o}$;

- if $P \stackrel{C}{\rightarrow}{ }_{I P O} P^{\prime}$ then $C[Q] \rightsquigarrow Q^{\prime}$ and $P^{\prime} \mathcal{R} Q^{\prime}$.

Barbed semi-saturated bisimilarity $\sim^{B S S}$ is the largest barbed semi-saturated bisimulation.

Proposition 3. In a reactive system having redex-IPOs, $\sim^{B S S}=\sim^{B S}$.

Reasoning on $\sim^{B S S}$ is easier than on $\sim^{B S}$, because instead of looking at the reductions in all contexts, we consider only IPO-transitions. Even if barbs are still quantified over all contexts, for many formalisms (as for MAs) it is actually enough to check if $P \downarrow_{o}$ implies $Q \downarrow_{o}$, since this condition implies that $\forall C[-]$, if $C[P] \downarrow_{o}$ then $C[Q] \downarrow_{o}$. Barbs satisfying this property are called contextual barbs.

Definition 12 (Contextual Barbs). A barb o is a contextual barb if whenever $P \downarrow_{o}$ implies $Q \downarrow_{o}$ then $\forall C[-], C[P] \downarrow_{o}$ implies $C[Q] \downarrow_{o}$.

\subsection{Weak Barbed Saturated Bisimilarity}

This section introduces weak barbed (semi-)saturated bisimilarity. We begin by recalling weak barbs. A state $P$ satisfies the weak barb $o$ (written $P \Downarrow_{o}$ ) if there exists a state $P^{\prime}$ such that $P \rightsquigarrow{ }^{*} P^{\prime}$ and $P^{\prime} \downarrow_{0}$.

Definition 13 (Weak Barbed Saturated Bisimulation). A symmetric relation $\mathcal{R}$ is a weak barbed saturated bisimulation if whenever $P \mathcal{R} Q$ then $\forall C[-]$

- if $C[P] \Downarrow_{o}$ then $C[Q] \Downarrow_{o}$;

- if $C[P] \rightsquigarrow^{*} P^{\prime}$ then $C[Q] \rightsquigarrow * Q^{\prime}$ and $P^{\prime} \mathcal{R} Q^{\prime}$.

Weak barbed saturated bisimilarity $\sim^{W B S}$ is the largest weak barbed saturated bisimulation. 
Definition 14 (Weak Barbed Semi-Saturated Bisimulation). A symmetric relation $\mathcal{R}$ is a weak barbed semi-saturated bisimulation if whenever $P \mathcal{R} Q$ then

$-\forall C[-]$, if $C[P] \downarrow_{o}$ then $C[Q] \Downarrow_{o}$;

- if $P \stackrel{C}{\rightarrow}[-]$ IPO $P^{\prime}$ then $C[Q] \rightsquigarrow * Q^{\prime}$ and $P^{\prime} \mathcal{R} Q^{\prime}$.

Weak barbed semi-saturated bisimilarity $\sim^{W B S S}$ is the largest weak barbed semisaturated bisimulation.

Proposition 4. In a reactive system having redex-IPOs, $\sim W B S S=\sim^{W B S}$.

Now we introduce weak contextual barbs. Analogously to the strong case, for those formalisms whose barbs are weakly contextual the first condition of Definition 14 becomes simpler: indeed, it suffices to check if $P \downarrow_{o}$ implies $Q \Downarrow_{o}$.

Definition 15 (Weak Contextual Barbs). $A$ barb o is a weak contextual barb if whenever $P \downarrow_{o}$ implies $Q \Downarrow_{o}$ then $\forall C[-], C[P] \downarrow_{o}$ implies $C[Q] \Downarrow_{o}$.

\section{Labelled Characterizations of Barbed Congruences}

This section proposes a labelled characterization of both strong and weak reduction barbed congruence for MAs, presented in Section 3. Indeed, MAs can be seen as a reactive system, with pure processes (up-to structural congruence) as ground terms: as shown in [10], pure processes must first be encoded into graphs, and the reduction semantics simulated by graph rewriting. We can then apply the borrowed contexts technique for distilling IPOs, which is proved to be an instance of the reactive system construction. The resulting ITS is the one that we presented in Section 4. Therefore, we can apply the notions of (weak) barbed saturated and semi-saturated bisimilarities, shown in the previous section, in order to capture those two behavioural semantics of MAs.

The first step is stated by the proposition below.

Proposition 5. Strong reduction barbed congruence over MAs $\cong$ coincides with barbed saturated bisimilarity $\sim^{B S}$. Similarly, weak reduction barbed congruence over $M A s \cong W$ coincides with weak barbed saturated bisimilarity $\sim^{W B S}$.

As shown in Section 5, we can efficiently characterize (weak) barbed saturated bisimilarity through the IPO-transition system, and the semi-saturated game. We can then characterize strong and weak reduction barbed congruence over MAs by instantiating Definitions 10 and 14, respectively, with the ITS $\mathcal{S}_{\mathcal{I}}$.

Moreover, the quantification over all contexts can be removed from the first condition of the definition of (semi-)saturated bisimulation.

Proposition 6. MAs barbs are both strong and weak contextual barbs.

We then obtain a simpler definition of (weak) semi-saturated bisimilarity. 
Definition 16 (Barbed Semi-Saturated Bisimulations for MAs). A symmetric relation $\mathcal{R}$ is a barbed semi-saturated bisimulation for $M A$ s if whenever $P \mathcal{R} Q$ then

- if $P \downarrow_{n}$ then $Q \downarrow_{n}$;

- if $P \stackrel{C[-]}{\longrightarrow} \mathcal{S}_{\mathcal{I}} P^{\prime}$ then $C[Q] \rightsquigarrow Q^{\prime}$ and $P^{\prime} \mathcal{R} Q^{\prime}$.

Barbed semi-saturated bisimilarity $\sim_{M A}^{B S S}$ is the largest barbed semi-saturated bisimulation.

A symmetric relation $\mathcal{R}$ is a weak barbed semi-saturated bisimulation for $M A s$ if whenever $P \mathcal{R} Q$ then

- if $P \downarrow_{n}$ then $Q \Downarrow_{n}$;

- if $P \stackrel{C[-]}{\longrightarrow} \mathcal{S}_{\mathcal{I}} P^{\prime}$ then $C[Q] \rightsquigarrow{ }^{*} Q^{\prime}$ and $P^{\prime} \mathcal{R} Q^{\prime}$.

Weak barbed semi-saturated bisimilarity $\sim_{M A}^{W B S S}$ is the largest weak barbed semisaturated bisimulation.

We finally introduce the main characterization theorem of the paper.

Theorem 3. Barbed semi-saturated bisimilarity for $M A s \sim_{M A}^{B S S}$ coincides with strong reduction barbed congruence $\cong$. Similarly, weak barbed semi-saturated bisimilarity $\sim_{M A}^{W B S S}$ coincides with weak reduction barbed congruence $\cong W$.

It is easy to note that the two statements of the theorem above follow from Proposition 5, and from Proposition 3 and 4 respectively.

\subsection{On Observing Ambient Migration}

An alternative labelled characterization of weak reduction barbed congruence is presented in 12 by Merro and Zappa Nardelli. However, the bisimulation that they propose is not defined in the standard way. They indeed note that in MAs the ability of a (restricted) ambient to migrate is unobservable, therefore in order to take this phenomenon into account they propose a modification of the usual definition of bisimulation. On the contrary, Rathke and Sobociński use instead in 11 the ordinary bisimilarity for characterizing the strong reduction barbed congruence. However, they are forced to add a set of what they call Honda-Tokoro rules, in order to account for the same phenomenon about ambient migrations. We remark that in our proposal we are never able to observe migrations of private ambients, thanks to the use of semi-saturations: this is shown by the following example for the weak semi-saturated case.

Example 2. Let us consider the example below, originally proposed in [12], which illustrates two weak reduction barbed congruent processes

$$
P=(\nu n) n[\text { in } k .0] \quad \text { and } \quad Q=\mathbf{0}
$$


The two processes $P$ and $Q$ are distinguished by the standard weak equivalence over our LTS $\mathcal{S}_{\mathcal{I}}$, since $P$ can interact with a context $-\mid k[R]$, while $\mathbf{0}$ cannot. The weak barbed semi-saturated bisimulation instead does not observe the migration of the private ambient $n$. The transition $P \stackrel{-\mid k[R]}{\longrightarrow} \mathcal{S}_{\mathcal{I}}(\nu n) k[n[0] \mid R]$ is indeed matched by $0\left|k[R] \rightsquigarrow^{*} 0\right| k[R]$. Moreover, since $(\nu n) k[n[0] \mid R]$ and $0 \mid k[R]$ are weak barbed semi-saturated equivalent, also $P$ and $Q$ are so.

\section{Conclusions and Future Work}

The main issues of this paper have been the introduction of barbed bisimilarities in reactive systems, and their exploitation for recasting the semantics of MAs.

In particular, we proposed the novel notions of barbed and weak barbed saturated bisimilarity over reactive systems, showing that they can be efficiently characterized through the IPO-transition systems by employing the semi-saturated game. We applied the framework to MAs, proving that it can capture the strong and the weak reduction barbed congruence for MAs, proposed by Rathke and Sobociński [11, and by Merro and Zappa Nardelli [12, respectively.

We thus obtained a labelled characterization for these barbed congruences, exploiting the ITS previously proposed in [10]. However, as it is typical of the LTSs distilled by IPOs, its presentation is far from standard. Therefore, we proposed an alternative, yet equivalent presentation for our ITS using SOS rules, which simplifies our proofs and furthermore allows for an easier comparison between our proposal and that one presented by Rathke and Sobociński in [11.

We consider such a presentation a first step towards solving the problem of synthesizing a set of SOS rules for describing the ITS distilled from a reactive system. Indeed, we are quite pleased by the parametric structure of the rules that we obtained, and we hope that we will be able to lift it to the instance of RPO adopted in graph rewriting, namely, borrowed contexts [13.

Finally, as discussed in Section 6, we recall that an alternative, labelled characterization of the strong reduction barbed congruence is presented in 11. Rathke and Sobociński use there the standard bisimilarity to capture the congruence, but they are forced to add a set of Honda-Tokoro rules to deal with the unobservability of ambient migrations. Our solution instead accounts for this phenomenon by the use of the barbed semi-saturated bisimulation. It is true however that the proposal in 11 does not need any additional observation, while in our approach the choice of the right notion of barb is left to the ingenuity of the researcher. As a future work we would like to extend our solution by considering an automatically derived notion of barb, in the style of [23] and [24].

\section{References}

1. Leifer, J., Milner, R.: Deriving bisimulation congruences for reactive systems. In: Palamidessi, C. (ed.) CONCUR 2000. LNCS, vol. 1877, pp. 243-258. Springer, Heidelberg (2000)

2. Bonchi, F., König, B., Montanari, U.: Saturated semantics for reactive systems. In: Logic in Computer Science, pp. 69-80. IEEE Computer Society, Los Alamitos (2006) 
3. Bonchi, F.: Abstract Semantics by Observable Contexts. PhD thesis, Department of Informatics, University of Pisa (2008)

4. Milner, R.: Bigraphs for petri nets. In: Desel, J., Reisig, W., Rozenberg, G. (eds.) Lectures on Concurrency and Petri Nets. LNCS, vol. 3098, pp. 686-701. Springer, Heidelberg (2004)

5. Sassone, V., Sobociński, P.: A congruence for Petri nets. In: Petri Nets and Graph Transformation. ENTCS, vol. 127, pp. 107-120. Elsevier, Amsterdam (2005)

6. Milner, R.: Communication and Concurrency. Prentice-Hall, Englewood Cliffs (1989)

7. Milner, R.: Communicating and Mobile Systems: the $\pi$-Calculus. Cambridge University Press, Cambridge (1999)

8. Milner, R., Sangiorgi, D.: Barbed bisimulation. In: Kuich, W. (ed.) ICALP 1992. LNCS, vol. 623, pp. 685-695. Springer, Heidelberg (1992)

9. Cardelli, L., Gordon, A.: Mobile ambients. TCS 240(1), 177-213 (2000)

10. Bonchi, F., Gadducci, F., Monreale, G.V.: Labelled transitions for mobile ambients (as synthesized via a graphical encoding). In: Expressiveness in Concurrency. ENTCS. Elsevier, Amsterdam (forthcoming, 2008)

11. Rathke, J., Sobociński, P.: Deriving structural labelled transitions for mobile ambients. In: van Breugel, F., Chechik, M. (eds.) CONCUR 2008. LNCS, vol. 5201, pp. 462-476. Springer, Heidelberg (2008)

12. Merro, M., Zappa Nardelli, F.: Behavioral theory for mobile ambients. Journal of the ACM 52(6), 961-1023 (2005)

13. Ehrig, H., König, B.: Deriving bisimulation congruences in the DPO approach to graph rewriting with borrowed contexts. Mathematical Structures in Computer Science 16(6), 1133-1163 (2006)

14. Sassone, V., Sobociński, P.: Reactive systems over cospans. In: Logic in Computer Science, pp. 311-320. IEEE Computer Society, Los Alamitos (2005)

15. Plotkin, G.D.: A structural approach to operational semantics. Journal of Logic and Algebraic Programming 60-61, 17-139 (2004)

16. Milner, R.: Pure bigraphs: Structure and dynamics. Information and Computation 204(1), 60-122 (2006)

17. Bonchi, F., Gadducci, F., König, B.: Process bisimulation via a graphical encoding. In: Corradini, A., Ehrig, H., Montanari, U., Ribeiro, L., Rozenberg, G. (eds.) ICGT 2006. LNCS, vol. 4178, pp. 168-183. Springer, Heidelberg (2006)

18. Milner, R.: Local bigraphs and confluence: Two conjectures. In: Expressiveness in Concurrency. ENTCS, vol. 175, pp. 65-73. Elsevier, Amsterdam (2007)

19. Di Gianantonio, P., Honsel, F., Lenisa, M.: RPO, second-order contexts, and $\lambda$ calculus. In: Amadio, R. (ed.) FOSSACS 2008. LNCS, vol. 4962, pp. 334-349. Springer, Heidelberg (2008)

20. Jensen, O., Milner, R.: Bigraphs and transitions. In: Principles of Programming Languages, pp. 38-49. ACM Press, New York (2003)

21. Grohmann, D., Miculan, M.: Reactive systems over directed bigraphs. In: Caires, L., Vasconcelos, V.T. (eds.) CONCUR 2007. LNCS, vol. 4703, pp. 380-394. Springer, Heidelberg (2007)

22. Fournet, C., Gonthier, G.: A hierarchy of equivalences for asynchronous calculi. In: Larsen, K.G., Skyum, S., Winskel, G. (eds.) ICALP 1998. LNCS, vol. 1443, pp. 844-855. Springer, Heidelberg (1998)

23. Honda, K., Yoshida, N.: On reduction-based process semantics. TCS 151(2), 437486 (1995)

24. Rathke, J., Sassone, V., Sobocinski, P.: Semantic barbs and biorthogonality. In: Seidl, H. (ed.) FOSSACS 2007. LNCS, vol. 4423, pp. 302-316. Springer, Heidelberg (2007) 